\title{
The Presence of a Transporter-Induced Protein Binding Shift: A New Explanation for Protein-Facilitated Uptake and Improvement for In Vitro-In Vivo Extrapolation
}

\author{
Christine M. Bowman, Hideaki Okochi, and 느eslie Z. Benet \\ Department of Bioengineering and Therapeutic Sciences, University of California, San Francisco, California
}

Received December 11, 2018; accepted January 18, 2019

\section{ABSTRACT}

Accurately predicting hepatic clearance is an integral part of the drug-development process, and yet current in vitro to in vivo (IVIVE) extrapolation methods yield poor predictions, particularly for highly protein-bound transporter substrates. Explanations for error include inaccuracies in protein-binding measurements and the lack of recognition of protein-facilitated uptake, where both unbound and bound drug may be cleared, violating the principles of the widely accepted free drug theory. A new explanation for proteinfacilitated uptake is proposed here, called a transporter-induced protein binding shift. High-affinity binding to cell-membrane proteins may change the equilibrium of the nonspecific binding between drugs and plasma proteins, leading to greater cellular uptake and clearance than currently predicted. The uptake of two lower protein-binding organic anion transporting polypeptide substrates (pravastatin and rosuvastatin) and two higher binding substrates (atorvastatin and pitavastatin) were measured in rat hepatocytes in incubations with protein-free buffer versus $100 \%$ plasma. Decreased unbound $K_{m}$ values and increased intrinsic clearance values were seen in the plasma incubations for the highly bound compounds, supporting the new hypothesis and mitigating the IVIVE underprediction previously seen for highly bound transporter substrates.

\section{Introduction}

Accurately predicting fundamental pharmacokinetic properties, such as clearance, is crucial when trying to improve the lengthy and expensive drug-discovery and -development process (Thomas et al., 2016). Hepatic clearance, which is associated with hepatic bioavailability after oral dosing and elimination from the systemic circulation, is used both early in discovery for rank ordering compounds and later in development for determining first-in-human doses. In vitro-in vivo extrapolation (IVIVE) for clearance predictions is commonly used, but large errors have been found when using human or rat microsomes or hepatocytes (Bowman and Benet, 2016; Wood et al., 2017). Furthermore, predictions are thought to be poorest for compounds that are highly protein-bound and substrates of transporters (Soars et al., 2007).

Traditionally, intrinsic clearance $\left(C L_{\mathrm{int}}\right)$ is measured in protein-free buffer, and after applying physiologically based scaling factors, a model of hepatic disposition, such as the well stirred model, is used to predict hepatic clearance $\left(C L_{\mathrm{H}}\right)$. In vitro methods such as equilibrium dialysis, ultrafiltration, and ultracentrifugation (Bohnert and Gan, 2013) are used separately to determine the equilibrium fraction of unbound drug $(f u)$,

C.M.B. was supported in part by the Pharmaceutical Research and Manufacturers of America Foundation Predoctoral Fellowship in Pharmaceutics and the National Science Foundation Graduate Research Fellowship Program [Grant 1144247]; L.Z.B. is a member of the University of California San Francisco Liver Center and supported by the National Institutes of Health [Grant P30 DK026743].

https://doi.org/10.1124/dmd.118.085779. which according to free drug theory (FDT) is what is available for metabolism (Trainor, 2007) and a parameter also included in the well stirred model; however, there is still a lack of confidence in measured $f u$ values as reflected in recent drug-drug interaction guidelines stating that the lower limit should be 0.01 regardless of actual measured values (Di et al., 2017). To try to reduce the uncertainty introduced with separately measuring $f u$, some groups have started using plasma in incubations (Shibata et al., 2000, 2002).

These investigations with plasma led to decreased unbound $\mathrm{Km}$ $(\mathrm{Km}, \mathrm{u})$ and increased $C L_{\text {int }}$ values compared with those generated in protein-free buffer (Blanchard et al., 2004, 2006), supporting the concept of protein-facilitated uptake, where highly bound ligands have more efficient hepatic uptake than can be accounted for by solely their unbound concentrations (Forker and Luxon, 1981, 1983; Weisiger and Ma, 1987; Tsao et al., 1988). The hypotheses that were proposed to explain how bound concentrations may also be involved were recently reviewed (Poulin et al., 2016; Bowman and Benet, 2018) and include the presence of a specific albumin receptor on the hepatocyte cell surface, the rate-limiting dissociation of ligand from the protein-ligand complex, the rate-limiting diffusion of ligand through the unstirred water layer, and interactions with the hepatocyte cell surface. Many of these previous hypotheses were suggested before hepatic transporters were recognized, and here a new hypothesis is proposed called a transporter-induced protein binding shift (TIPBS), a term and concept first suggested by Baik and Huang (2015).

As the transporter field has evolved, exceptions to the FDT have emerged, and it is now known that uptake transporters, such as organic

ABBREVIATIONS: ACN, acetonitrile; ANS, 1-anilino-8-naphthalene sufonate; $C L_{\mathrm{H}}$, hepatic clearance; $C L_{\text {int }}$, intrinsic clearance; FDT, free drug theory; $f u_{\mathrm{p}}$, fraction unbound in plasma; HSA, human serum albumin; IVIVE, in vitro to in vivo extrapolation; $K_{\mathrm{m}, \mathrm{u}}$, unbound $K_{\mathrm{m}}$; LC-MS/MS, chromatography-tandem mass spectrometry; OATP, organic anion transporting polypeptide; $P_{\text {dif, }}$, unbound passive diffusion; TIPBS, transporterinduced protein binding shift. 


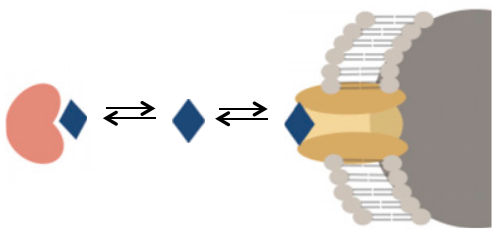

B

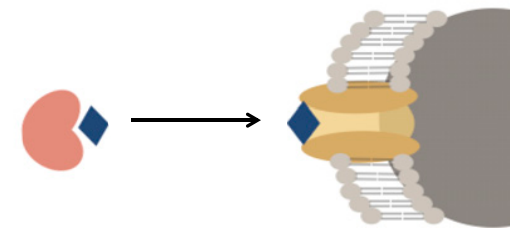

Fig. 1. The traditional view of drug dissociating from plasma proteins and being at equilibrium before uptake is depicted in (A). The concept of a TIPBS is depicted in (B) where high-affinity binding to transporters may strip the drug directly from the proteins before equilibrium is reached. anion transporting polypeptides (OATPs), are able to control a drug's access to hepatocytes and increase the intracellular free concentration significantly above that in plasma (Giacomini et al., 2010). With the TIPBS hypothesis, high-affinity binding to such transporters may be able to change the equilibrium of the nonspecific binding between a drug and plasma protein. If a highly protein-bound drug has a higher affinity for a transporter than for the plasma protein, the transporter may be able to strip the drug directly from the protein before the drug dissociates itself and is at binding equilibrium (Fig. 1B). In this case, protein binding would not be limiting the access of these compounds, and using $f u$ values measured at equilibrium in vitro would be inaccurate. Using rat hepatocytes and statins, known OATP1B1 transporter substrates, we show that there is an increase in measured affinity [a decrease in unbound $K_{\mathrm{m}}$ ] for uptake in $100 \%$ human plasma versus protein-free buffer incubations with highly bound drugs and smaller changes in $K_{\mathrm{m}, \mathrm{u}}$ values for drugs with low binding, for which the transporter-induced shift would not occur.

The TIPBS hypothesis proposed here and alternative previous hypotheses, as we recently reviewed (Bowman and Benet, 2018), have been promulgated in an attempt to explain the observed discordance of protein-binding effects from the FDT. These hypotheses are proposed despite recognition that for a simple donor compartment-receiver compartment diffusion model, the permeability surface product for passive diffusion of unbound drug across the membrane should be independent of the presence or absence of plasma protein.

\section{Materials and Methods}

Materials. Atorvastatin was purchased from TCI America (Portland, OR), pitavastatin from ApexBio (Houston, TX), rosuvastatin from Toronto Research Chemicals (North York, ON, Canada), and [3H(G)] pravastatin sodium salt (specific activity, $5 \mathrm{Ci} / \mathrm{mmol}$ ) from American Radiolabeled Compounds (St. Louis, MO). Mixed-gender pooled human plasma was purchased from Biologic Specialty Corporation. Male Sprague-Dawley rats (250-270 g) were purchased from Charles River Laboratories (Wilmington, MA).

Hepatocyte Isolation. Rat hepatocytes were isolated using a modified collagenase perfusion method as previously described (Lam and Benet, 2004; Lam et al., 2006). Briefly, the rats were given an i.p. injection of $1 \mathrm{ml} / \mathrm{kg}$ ketamine/ xylazine $(91 \mathrm{mg} / \mathrm{ml} ; 9 \mathrm{mg} / \mathrm{ml})$ before surgery. The portal vein was cannulated with an i.v. catheter (BD Biosciences, San Jose, CA) and perfused with oxygenated liver perfusion medium (Gibco/Thermo Fisher, Waltham, MA) for 10 minutes, followed by perfusion with oxygenated liver perfusion medium supplemented with $1.2 \mathrm{U} / \mathrm{ml}$ collagenase (Sigma-Aldrich, St. Louis, MO) for 10 minutes at $20 \mathrm{ml} / \mathrm{min}$. The digested livers were excised and broken down by gentle tapping with a glass stirring rod. Cells were washed with ice-cold hepatocyte wash medium (Gibco/Thermo Fisher) and centrifuged at $50 \mathrm{~g}$ for 3 minutes. Hepatocytes were separated by $44 \%$ Percoll (Sigma-Aldrich) in hepatocyte wash medium and centrifuged at $250 \mathrm{~g}$ for 10 minutes at $4^{\circ} \mathrm{C}$. Cell viability was determined using the trypan blue exclusion method, and cells with viability $>90 \%$ were used for uptake studies.

Hepatocyte Uptake Studies. Hepatocyte suspensions of either protein-free Krebs-Henseleit buffer ( $\mathrm{pH} 7.4$ ) or $100 \%$ plasma were preincubated at $37^{\circ} \mathrm{C}$ in 24-well plates for 10 minutes. Uptake studies were done once for each substrate and condition in triplicate and were initiated by adding various concentrations of drug solutions (1.0-100 $\mu \mathrm{M}$ for atorvastatin; $0.05-100 \mu \mathrm{M}$ for pitavastatin; $0.1-$ $300 \mu \mathrm{M}$ for pravastatin; and $0.05-100 \mu \mathrm{M}$ for rosuvastatin) to the hepatocyte suspensions. After 1 minute for atorvastatin, pitavastatin, and rosuvastatin and 2 minutes for pravastatin (based on time-course results not shown here), reactions were terminated by transferring 0.5 million hepatocytes $(1 \mathrm{ml}$ of the shaken mixture of $800 \mu \mathrm{l}$ of one million cells $/ \mathrm{ml}$ and $800 \mu \mathrm{l}$ of drug solution) into a centrifuge tube containing $300 \mu \mathrm{l}$ of a mixture of mineral and silicone oil (density $=1.015$ ) and centrifuging at $13,000 \mathrm{~g}$ for 10 seconds. After removing the drug solutions and oil layers by pipetting, the pravastatin cell pellets were resuspended in $200 \mu \mathrm{l}$ of scintillation cocktail and sonicated to ensure complete cell lysis. Intracellular concentration was measured using a scintillation counter (LS6000TA; Beckman Coulter, Fullerton, CA). For atorvastatin, pitavastatin, and rosuvastatin, cell pellets were resuspended in $200 \mu \mathrm{l}$ of water and sonicated. Methanol and acetonitrile ( $\mathrm{ACN}$ ) containing internal standard and $3 \%$ formic acid were subsequently added to samples (1:1:2; sample:methanol:ACN) to precipitate the protein. After centrifuging at 13,000g for 10 minutes, the supernatants were transferred into high-performance liquid chromatography vials for liquid chromatography-tandem mass spectrometry (LC-MS/MS) analysis. Measuring cold pravastatin uptake with LC-MS/MS was attempted; however, two inseparable peaks appeared with the methods.

LC-MS/MS Analysis. All samples were analyzed using a Shimadzu (Carlsbad, CA) high-performance liquid chromatography binary pump system coupled to a Sciex (Foster City, CA) API 4000 triple-quadrupole tandem mass spectrometer. The TurbolonSpray voltage was set at $5500 \mathrm{~V}$ and operated in positive electrospray ionization mode. The LC separations were done using a Zorbax (Agilent, Santa Clara, CA) C8 column $(3.5 \mu \mathrm{m}, 4.6 \times 50 \mathrm{~mm})$, and the mobile phases consisted of water with $10 \mathrm{mM}$ ammonium acetate (mobile phase A) and ACN with $10 \mathrm{mM}$ ammonium acetate (mobile phase B). A gradient elution with a flow rate of $0.7 \mathrm{ml} / \mathrm{min}$ was used where $35 \%$ B increased linearly to $80 \%$ at 3 minutes, at which point it was increased to $95 \%$ until 3.75 minutes, and then decreased to $35 \%$ and equilibrated until the end of the run at 5 minutes. The following transitions were measured $(\mathrm{Q} 1>\mathrm{Q} 3)$ : atorvastatin $(559.0>$ $440.2)$, pitavastatin $(422.4>290.3)$, rosuvastatin $(482.3>258.3)$, and the internal standard tolbutamide $(271.1>172.1)$.

To test for potential matrix effects with atorvastatin, pitavastatin, and rosuvastatin, calibration curves were created using stock solutions spiked into protein-free buffer and spiked into sonicated hepatocytes that had been incubated in protein-free buffer and $100 \%$ plasma. Process efficiency (comparison between spiked samples vs. neat solutions) was $95.9 \%-107 \%$ for atorvastatin, $101 \%-111 \%$ for pitavastatin, and $88.6 \%-108 \%$ for rosuvastatin. The matrix effect was considered minimal. The lower limits of quantitation were 50, 0.5, and $5 \mathrm{nM}$ for atorvastatin, pitavastatin, and rosuvastatin, respectively, and the average recoveries were $102 \% \pm 7 \%, 100 \% \pm 3 \%$, and $98.2 \% \pm 8 \%$, for atorvastatin, pitavastatin, and rosuvastatin, respectively.

For the calibration curves used, stock solutions (minimum six concentrations) were added to protein-free buffer, and the same calibration curve was used for the buffer and plasma samples of each compound. The concentration range for atorvastatin was $50-4000 \mathrm{nM}\left(1 / x^{2}\right.$ weighting, $\left.r^{2}=0.99\right)$; for pitavastatin, $0.5-$ $1500 \mathrm{nM}\left(1 / x^{2}\right.$ weighting, $\left.r^{2}=0.99\right)$; and for rosuvastatin, $5-1500 \mathrm{nM}(1 / x$ weighting, $\left.r^{2}=0.99\right)$. Interday precision values (percent coefficient variation) were between $5.31 \%$ and $10.7 \%$ for atorvastatin, between $3.40 \%$ and $10.0 \%$ for pitavastatin, and between $2.41 \%$ and $15.3 \%$ for rosuvastatin; and interday accuracies (percent relative error) were between $-4.00 \%$ and $2.00 \%$ for atorvastatin, between $-2.90 \%$ and $2.40 \%$ for pitavastatin, and between $-1.20 \%$ and $0.667 \%$ for rosuvastatin.

Data Analysis. Data analyses were done using GraphPad Prism version 7 (GraphPad Software, La Jolla, CA). The total drug dosing concentrations were 
A
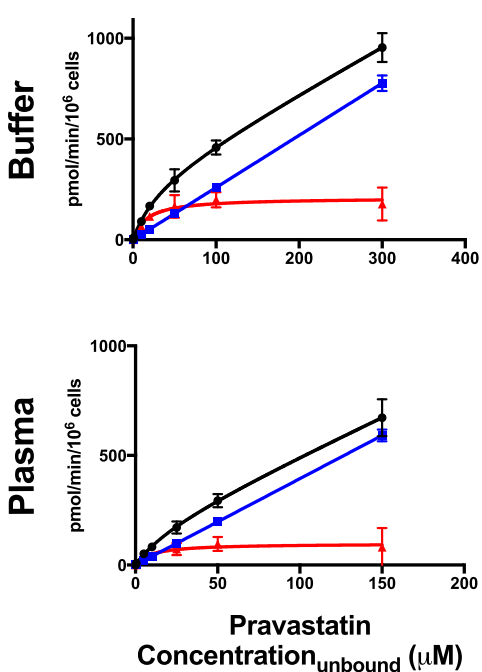

B
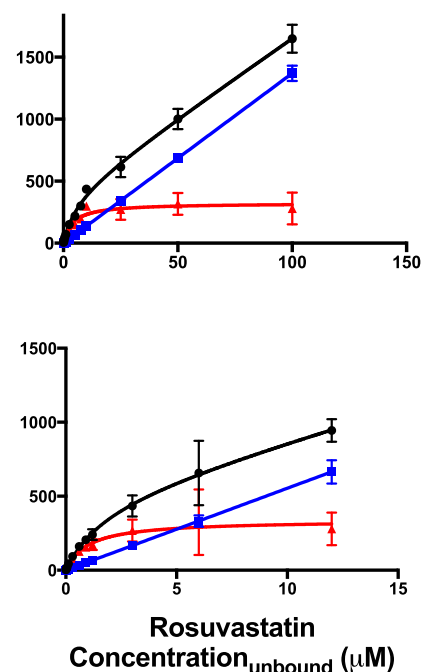

C
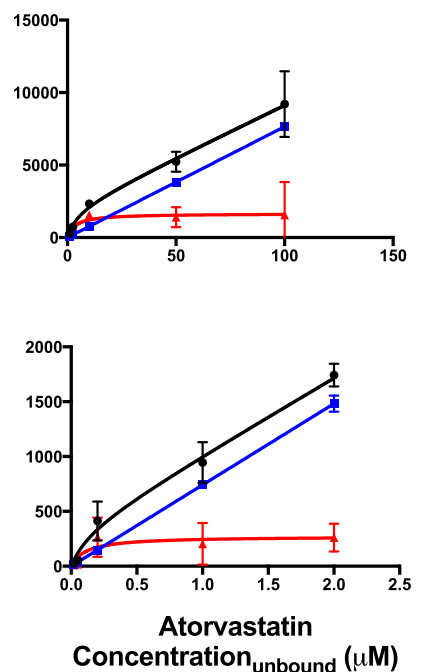

D
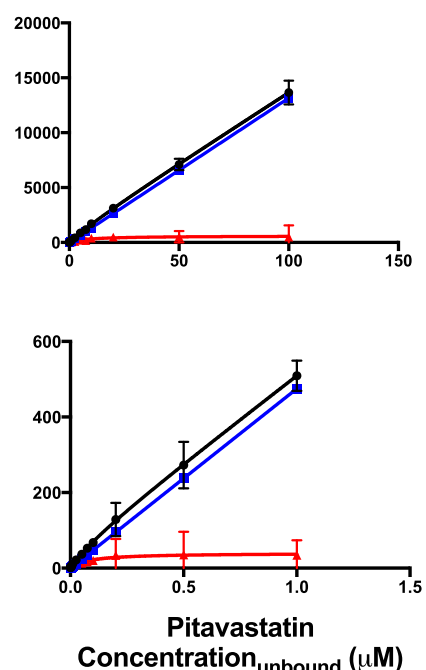

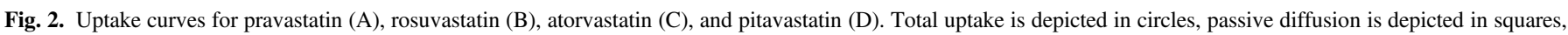
and active uptake is depicted in triangles. The error bars represent the S.D. of the triplicate.

corrected to unbound drug concentrations. For the protein-free buffer incubations, the fraction unbound $\left(f \mathrm{u}_{\mathrm{p}}\right)$ was assumed to be 1 ; so the unbound concentration was the same as total dosing concentration. For the plasma incubations, $f u_{\mathrm{p}}$ values in the following Food and Drug Administration labels were used: 0.020 for atorvastatin [Lipitor (atorvastatin calcium), 1996] 0.010 for pitavastatin [Livalo (pitavastatin), 2009], 0.50 for pravastatin [Pravachol (pravastatin sodium), 1991], 0.12 for rosuvastatin [Crestor (rosuvastatin calcium), 2003]

The kinetic parameters for uptake were estimated by fitting the intracellular concentrations to following equation: $v=\left(V_{\max } \times S\right) /\left(K_{m}+S\right)+P_{\text {dif }} \times S$, where $v$ is the rate of uptake (picomoles per minute per $10^{6}$ cells), $V_{\max }$ is the maximum uptake rate (picomoles per minute per $10^{6}$ cells), $S$ is the substrate concentration (micromolar), $K_{m}$ is the Michaelis-Menten constant (micromolar), and $P_{d i f}$ is the nonsaturable diffusion constant (microliters per minute per $10^{6}$ cells). The linear portion of the total uptake curve represents the passive diffusion, and the difference between the total uptake and passive diffusion represents the active transport.

\section{Results}

The uptake curves for the four known OATP substrates in both incubation conditions are shown in Fig. 2. As expected, all compounds exhibited both passive diffusion and active uptake. The calculated $K_{\mathrm{m}, \mathrm{u}}, V_{\mathrm{max}}, P_{\mathrm{dif,u}}$, and $C L_{\text {int }}$ values are reported in Table 1 . The $K_{\mathrm{m}, \mathrm{u}}$ values generated in the protein-free buffer aligned well with previously reported values in the literature. The values generated in the plasma incubations were similar to those in the buffer for the lower protein-binding compounds ( 9.66 vs. $16.5 \mu \mathrm{M}$ for pravastatin and 0.995 vs. 4.00 for rosuvastatin). There were much larger $\mathrm{K}_{\mathrm{m}, \mathrm{u}}$ differences for the highly bound compounds where the apparent values were $31.4-$ and 107 -fold lower in the plasma incubations for atorvastatin and pitavastatin, respectively (Fig. 3A).

The $V_{\max }$ values showed a similar, but less marked, trend (Fig. 3B). Values generated in the two incubations were similar for pravastatin (2.12-fold different) and rosuvastatin (0.953-fold different), whereas the decreases in apparent $V_{\max }$ were greater in the plasma for atorvastatin (6.07-fold lower) and pitavastatin (15.0-fold lower). These decreases in $V_{\text {max }}$ were less than the decreases in $K_{\mathrm{m}, \mathrm{u}}$, so when $C L_{\mathrm{int}}\left(V_{\mathrm{max}} / K_{\mathrm{m}, \mathrm{u}}\right)$ was examined, there were increases for the higher binding compounds. Figure $3 \mathrm{C}$ shows that as the fraction unbound decreases, the difference in $C L_{\text {int }}$ (plasma/buffer) increases.

TABLE 1

$K_{\mathrm{m}, \mathrm{u}}, V_{\mathrm{max}}, P_{\mathrm{dif}, \mathrm{u}}$, and $C L_{\text {int }}$ values generated for each compound in buffer and plasma incubations

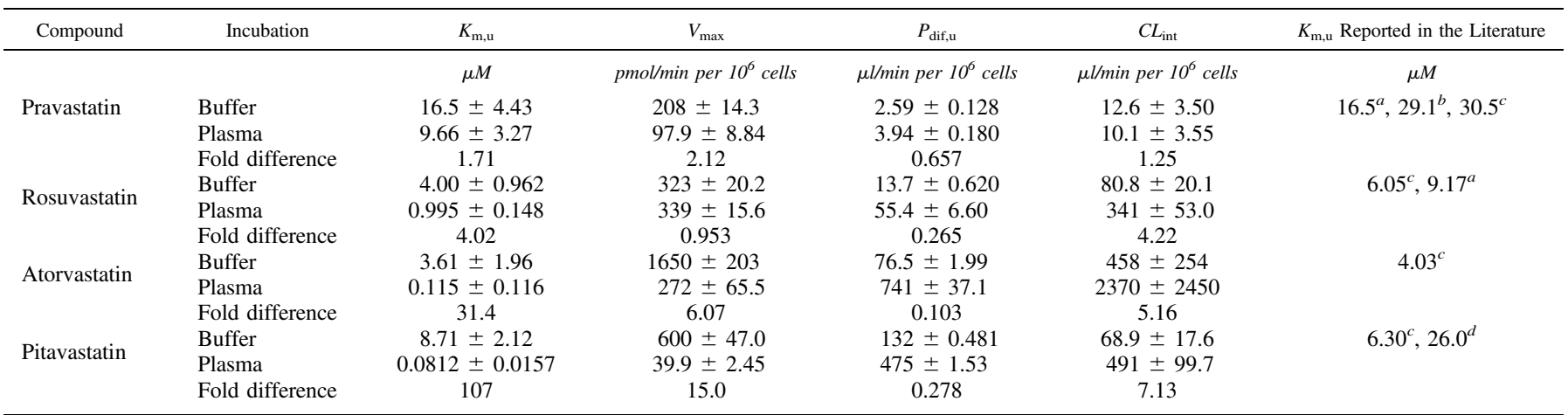

${ }^{a}$ Nezasa et al. (2003).

${ }^{b}$ Yamazaki et al. (1993).

${ }^{c}$ Yabe et al. (2011).

${ }^{d}$ Shimada et al. (2003) 

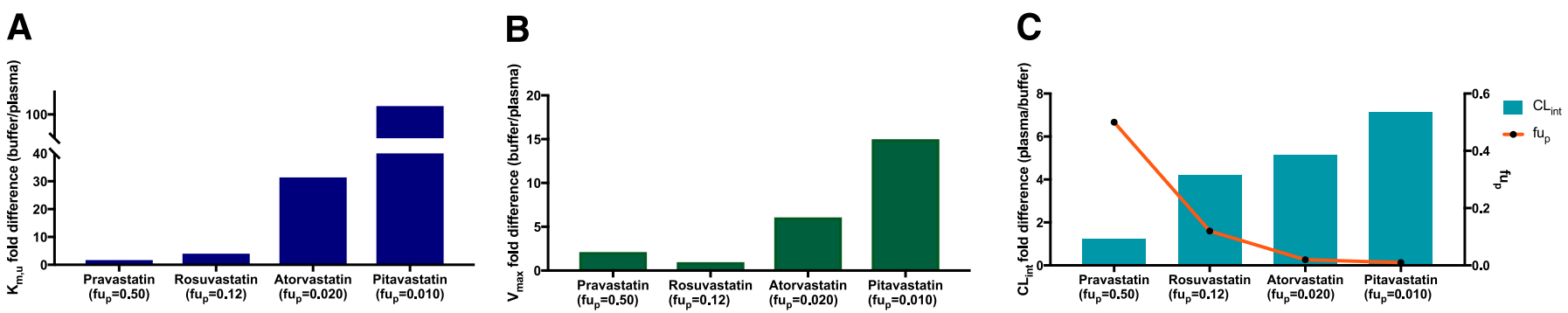

Fig. 3. The fold difference in $K_{\mathrm{m}, \mathrm{u}}$ values (buffer/plasma) (A), $V_{\max }$ values (buffer/plasma) (B), and $C L_{\text {int }}$ values (plasma/buffer) (C) between the two incubations.

\section{Discussion}

Obtaining accurate in vitro data is crucial for $C L_{\mathrm{H}}$ predictions; however, there are often larger errors for compounds that are substrates of transporters (Soars et al., 2007). A previous study examining the active uptake of seven OATP substrates with sandwich culture human hepatocytes in protein-free incubations found that predictions were poorest for highly protein-bound substrates, whereas pravastatin and rosuvastatin, compounds with the lowest protein-binding, gave more accurate predictions (Jones et al., 2012).

Considering this trend, we proposed the idea of a transporter-induced protein-binding shift, where high-affinity binding to transporters may strip ligands directly from plasma proteins before they dissociate. For OATP substrates with lower protein binding, such as pravastatin and rosuvastatin, where there is already free drug near the uptake transporters, such a shift may not occur, and current methodologies may yield accurate IVIVE predictions; however, for transporter substrates with high binding, such as pitavastatin and atorvastatin, where TIPBS would occur, current equilibrium protein-binding measurements may be driving the high IVIVE error.

Here, the uptake of four statins, known to be OATP substrates (Kalliokoski and Niemi, 2009), were measured in rat hepatocytes with protein-free buffer and $100 \%$ human plasma. Given the well-known human $\mathrm{fu}_{\mathrm{p}}$ values for these compounds, $100 \%$ human plasma was used. With the frequent similarity between human and rat $f u_{\mathrm{p}}$ values (Hosea et al., 2009; Colclough et al., 2014), similar results would be expected if $100 \%$ rat plasma were used. For the low-binding compounds, pravastatin and rosuvastatin, $K_{\mathrm{m}, \mathrm{u}}$ values were similar in the two incubations (1.71- and 4.02-fold different, respectively), whereas the difference in the values increased with increased protein binding for atorvastatin and pitavastatin (31.4- and 107-fold different, respectively). If protein-binding is not limiting the uptake of atorvastatin and pitavastatin, a lower apparent $K_{\mathrm{m}, \mathrm{u}}$ value (or increased affinity) would be expected when adding plasma to hepatocyte incubations as compared with using protein-free buffer. TIPBS can be viewed mechanistically, perhaps considering the competing processes as competitive inhibition (Fig. 4). The traditional enzyme, substrate, and inhibitor (Fig. 4A) are swapped out for drug, transporter, and protein (Fig. 4B), where, according to FDT, the protein would be "inhibiting" the access of the drug to the transporter. With TIPBS and the noninhibitory protein, a larger $k_{1}$ is expected, resulting in decreased $K_{\mathrm{m}, \mathrm{u}}$ values.

This study also noted greater decreases in $V_{\max }$ values for the highly bound compounds. Pravastatin and rosuvastatin had 2.12- and 0.953fold differences in plasma compared with buffer, whereas atorvastatin and pitavastatin had differences of 6.07 and 15.0. Despite these larger decreases, $K_{\mathrm{m}, \mathrm{u}}$ decreases were larger, leading to increased $C L_{\mathrm{int}}$ values for atorvastatin and pitavastatin. The TIPBS hypothesis and these larger generated $C L_{\text {int }}$ values can explain and mitigate the IVIVE underprediction seen for highly bound transporter substrates. A larger $C L_{\text {int }}$ increase would have been expected for atorvastatin given that the 5.16-fold increase is only marginally larger than the 4.22-fold increase seen with the lower binding rosuvastatin; however, since atorvastatin is the most lipophilic of the four compounds, perhaps the active uptake process is not as crucial, and TIPBS should have less impact.

Similar protein-facilitated uptake has been previously noted; however, alternative hypotheses are frequently cited. The potential role of the hepatocyte cell surface is often considered, in contrast to the role of high-affinity transporters described here. One such previous proposal was that ionic interactions between the albumin-drug complex and the hepatocyte plasma membrane may decrease the diffusional distance for unbound ligand (Burczynski et al., 1997); another proposal stated that binding of the albumin-drug complex to the cell surface may lead to a conformational change in albumin, enhancing drug dissociation (Tsao et al., 1988); however, despite citing these alternative hypotheses, recent data in the literature agree with the TIPBS concept.

Miyauchi et al. (2018) examined the uptake of 1-anilino-8-naphthalene sufonate (ANS) in rat hepatocytes with bovine serum albumin and the uptake of pitavastatin in human hepatocytes with human serum albumin (HSA). Although the addition of protein led to increases in unbound uptake clearances for both compounds, the difference was greater for pitavastatin, which is known to have high affinity for OATP, whereas ANS has a relatively lower affinity for Oatp. Kim et al. (2019) examined the uptake of 11 OATP substrates with varying concentrations of HSA. Similar to our results, pravastatin uptake did not change with the addition of HSA, and using their previously proposed facilitated-dissociation

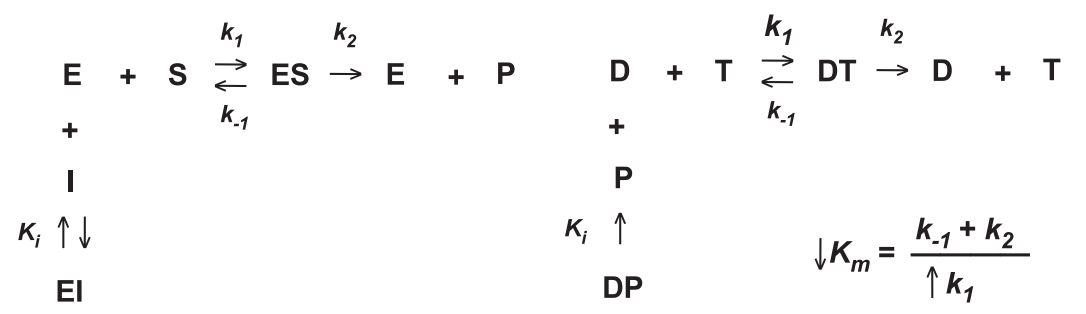

Fig. 4. A traditional schematic of competitive inhibition with enzyme (E), inhibitor $(\mathrm{I})$, substrate $(\mathrm{S})$, and product $(\mathrm{P})$ is shown in (A). A modified version where protein $(\mathrm{P})$ acts as an inhibitor for drug (D) to access the transporter (T) is shown in (B). With a TIPBS, the transporter will strip the drug from the DP complex, leading to a larger $k_{1}$ and lower $K_{\mathrm{m}}$. 
TABLE 2

Data from Poulin et al. (2017) and Bounakta et al. (2018) also supporting the trends seen with a transporter-induced protein binding shift

\begin{tabular}{|c|c|c|c|c|c|c|c|c|}
\hline Compound & $f u_{\mathrm{p}}$ & $\begin{array}{c}K_{\mathrm{m}} \\
{[\mathrm{ALB}]=} \\
0 \mathrm{~g} / \text { liter }\end{array}$ & $\begin{array}{l}K_{\mathrm{m}}[\mathrm{ALB}]= \\
30 \mathrm{~g} / \mathrm{l}\end{array}$ & $\begin{aligned} K_{\mathrm{m}, \mathrm{u}}= & K_{\mathrm{m}} \cdot f u_{\mathrm{p}}[\mathrm{ALB}]= \\
& 30 \mathrm{~g} / \text { liter }\end{aligned}$ & $\begin{array}{l}K_{\mathrm{m}, \mathrm{u}} \text { Fold } \\
\text { Difference }\end{array}$ & $\begin{array}{c}V_{\max }(\mathrm{nmol} / \text { min per mg })[\mathrm{ALB}]= \\
0 \mathrm{~g} / \mathrm{liter}\end{array}$ & $\begin{array}{c}V_{\max }()[\mathrm{ALB}]= \\
30 \mathrm{~g} / \mathrm{liyer}\end{array}$ & $\begin{array}{l}V_{\max } \text { Fold } \\
\text { Difference }\end{array}$ \\
\hline & & $\mu M$ & $\mu M$ & & & $\mathrm{nmol} / \mathrm{min}$ per $\mathrm{mg}$ & nmol/min per $\mathrm{mg}$ & \\
\hline Bisphenol A & 0.045 & 13.4 & 3.5 & 0.16 & 83.8 & 8.0 & 1.3 & 6.2 \\
\hline Naproxen & 0.12 & 98.9 & 174.4 & 20.9 & 4.73 & 2.9 & 2.2 & 1.3 \\
\hline
\end{tabular}

kinetic model, the uptake of rosuvastatin with 5\% HSA was estimated to increase by 2.48 -fold. Uptake of the remaining compounds, all with high protein binding, was estimated to increase to a greater extent, up to 63.8-fold with valsartan, except pitavastatin (a 2.44-fold increase was predicted). Their model predicts the contribution of albumin-mediated uptake to be similar for both pitavastatin and rosuvastatin $(56.9 \%$ and $54.4 \%$, respectively).

Both articles stated that, "the higher the affinity for the transporter, the more effective is the albumin-mediated enhancement," agreeing with the concept of TIPBS. Additional previous support for TIPBS was seen when measuring the uptake of a highly bound new chemical entity, shown to be an OATP1B3 substrate. $C L_{\text {int }}$ increased with increased HSA concentration in transfected human embryonic kidney (HEK293) cells, where the mechanisms hypothesized with the hepatocyte cell surface may not be present, but overexpression of the OATP transporter is present (Fukuchi et al., 2017).

Examining the pitavastatin data, Miyauchi et al. (2018) calculated the dissociation constant $\left(K_{\mathrm{d}}\right)$ of bound albumin from the hepatocyte cell surface to be $199 \pm 61 \mu \mathrm{M}$ with the facilitated-dissociation model (Tsao et al., 1988) and $275 \pm 131 \mu \mathrm{M}$ with a kinetic model proposed by Forker and Luxon (1983). Simultaneously fitting data from 10 OATP substrates, including pitavastatin, Kim et al. (2019) calculated the value to be $45.2 \pm 13.0 \mu \mathrm{M}$. Using fluorescence quenching, Shi et al. (2017) measured the binding constant of pitavastatin to bovine serum albumin as $0.56 \times 10^{4} \mathrm{M}^{-1}$ at $310 \mathrm{~K}$; inverting this, the dissociation constant would be $179 \mu \mathrm{M}$. Although not directly comparable, as $K_{\mathrm{d}}$ is a thermodynamic constant, whereas $K_{\mathrm{m}}$ is a kinetic constant, in this study the measured $K_{\mathrm{m} . \mathrm{u}}$ values for the interaction between pitavastatin and active uptake transporters were $17.3 \mu \mathrm{M}$ in buffer and $0.0688 \mu \mathrm{M}$ in plasma, which are significantly lower than the $K_{\mathrm{d}}$ values for binding to the hepatocyte surface or to protein.

The yet-to-be explained decrease in $V_{\max }$ values in plasma incubations for highly bound compounds has been previously noted. Based on the idea of competitive inhibition with TIPBS (Fig. 4B), the $V_{\max }$ values were expected to remain the same across incubations and across $f u$ ranges. As described by Poulin et al. (2017) and Bounakta et al. (2018), the clearance of bisphenol A and naproxen were measured using isolated perfused rat livers with and without albumin. Bisphenol A, the higher binding compound, had an 83.8 -fold decrease in its apparent $K_{\mathrm{m}, \mathrm{u}}$ value with albumin addition (compared with the 4.73 -fold decrease for the lower binding naproxen), and the $V_{\max }$ value of bisphenol A decreased 6.2-fold with albumin (while decreasing 1.3-fold for naproxen) (Table 2). A potential explanation could be that a weaker attraction, mentioned earlier, between the protein-drug complex and the hepatocyte cell surface brings more bound drug near the cell, and once the complex is oriented correctly, the transporter with higher affinity can strip the drug directly from the protein. The additional larger protein-drug complexes near the surface may limit access of both bound and free drug to transporters, decreasing the maximum velocity of substrate transport compared with protein-free incubations.

In summary, the $K_{\mathrm{m}, \mathrm{u}}$ decrease and $C L_{\mathrm{int}}$ increase for the highly protein-bound statins support the idea of a transporter-induced protein binding shift and suggest that plasma should be used in hepatocyte incubations for highly bound transporter substrates. TIPBS can also explain some of the large IVIVE error seen for highly bound compounds. If protein binding is not restricting the access of highly bound transporter substrates, perhaps total concentration, not unbound concentration, should be used in clearance predictions, an idea previously applied for alternate reasons (Obach, 1999; Poulin et al., 2012). To further support this hypothesis, additional studies are needed to determine the off-rate of drug dissociating from plasma protein versus the on-rate of drug associating with the membrane transporters under the two incubation conditions and studies to differentiate transportermediated uptake versus transporter binding.

It is important to note that the proposed shift may occur with any type of plasma protein and may occur anywhere in the body where there is interplay between plasma proteins, cells, and transporters, including the intestine and brain. That is, the shift is not specific to albumin and hepatocytes only. Previous studies have found facilitated uptake with $\beta$-lactoglobulin (Nunes et al., 1988; Burczynski et al., 1990) and ligandin (Stollman et al., 1983), as well as with myocytes (Hütter et al., 1984a,b; Rauch et al., 1987; Sorrentino et al., 1989; Elmadhoun et al., 2001), adipocytes (Sorrentino et al., 1989), proximal tubules (Besseghir et al., 1989), perfused kidney (Taft and Sweeney, 1995), and brain (Pardridge et al., 1983).

\section{Acknowledgments}

We thank Dr. Yong Huang for introducing to us the concept of transporter induced protein binding shift and for helpful discussions.

\section{Authorship Contributions}

Participated in research design: Bowman, Okochi, Benet.

Conducted experiments: Bowman, Okochi.

Performed data analysis: Bowman, Okochi, Benet.

Wrote or contributed to the writing of the manuscript: Bowman, Okochi, Benet.

\section{References}

Baik J and Huang Y (2015) Transporter-induced protein binding shift (TIPBS) hypothesis and modeling, in 20th North American ISSX Meeting. Orlando, Florida, October 2015.

Besseghir K, Mosig D, and Roch-Ramel F (1989) Facilitation by serum albumin of renal tubular secretion of organic anions. Am J Physiol 256:F475-F484.

Blanchard N, Hewitt NJ, Silber P, Jones H, Coassolo P, and Lavé T (2006) Prediction of hepatic clearance using cryopreserved human hepatocytes: a comparison of serum and serum-free incubations. J Pharm Pharmacol 58:633-641.

Blanchard N, Richert L, Notter B, Delobel F, David P, Coassolo P, and Lavé T (2004) Impact of serum on clearance predictions obtained from suspensions and primary cultures of rat hepatocytes. Eur J Pharm Sci 23:189-199.

Bohnert T and Gan LS (2013) Plasma protein binding: from discovery to development. J Pharm Sci 102:2953-2994.

Bounakta S, Bteich M, Mantha M, Poulin P, and Haddad S (2018) Predictions of bisphenol A hepatic clearance in the isolated perfused rat liver (IPRL): impact of albumin binding and of co-administration with naproxen. Xenobiotica 48:135-147.

Bowman CM and Benet LZ (2016) Hepatic clearance predictions from in vitro-in vivo extrapolation and the biopharmaceutics drug disposition classification system. Drug Metab Dispos 44: 1731-1735.

Bowman CM and Benet LZ (2018) An examination of protein binding and protein-facilitated uptake relating to in vitro-in vivo extrapolation. Eur J Pharm Sci 123:502-514.

Burczynski FJ, Moran JB, Cai Z-S, and Forker EL (1990) $\beta$-lactoglobulin enhances the uptake of free palmitate by hepatocyte monolayers: the relative importance of diffusion and facilitated dissociation. Can J Physiol Pharmacol 68:201-206. 
Burczynski FJ, Wang GQ, and Hnatowich M (1997) Effect of binding protein surface charge on palmitate uptake by hepatocyte suspensions. Br J Pharmacol 120:1215-1220.

Colclough N, Ruston L, Wood JM, and MacFaul PA (2014) Species differences in drug plasma protein binding. MedChemComm 5:963-967.

Crestor (rosuvastatin calcium). (2003) Package insert. AstraZeneca Pharmaceuticals LP, Wilmington, $\mathrm{DE}$

Di L, Breen C, Chambers R, Eckley ST, Fricke R, Ghosh A, Harradine P, Kalvass JC, Ho S, Lee $\mathrm{CA}$, et al. (2017) Industry perspective on contemporary protein-binding methodologies: considerations for regulatory drug-drug interaction and related guidelines on highly bound drugs. J Pharm Sci 106:3442-3452.

Elmadhoun BM, Wang GQ, Kirshenbaum LA, and Burczynski FJ (2001) Palmitate uptake by neonatal rat myocytes and hepatocytes. Role of extracellular protein. Eur J Biochem 268 $3145-3153$.

Forker EL and Luxon BA (1981) Albumin helps mediate removal of taurocholate by rat liver. $J$ Clin Invest 67:1517-1522.

Forker EL and Luxon BA (1983) Albumin-mediated transport of rose bengal by perfused rat liver. Kinetics of the reaction at the cell surface. $J$ Clin Invest 72:1764-1771.

Fukuchi Y, Toshimoto K, Mori T, Kakimoto K, Tobe Y, Sawada T, Asaumi R, Iwata T, Hashimoto Y, Nunoya K-I, et al. (2017) Analysis of nonlinear pharmacokinetics of a highly albumin-bound compound: contribution of albumin-mediated hepatic uptake mechanism. J Pharm Sci 106: 2704-2714.

Giacomini KM, Huang SM, Tweedie DJ, Benet LZ, Brouwer KL, Chu X, Dahlin A, Evers R, Fischer V, Hillgren KM, et al.; International Transporter Consortium (2010) Membrane transporters in drug development. Nat Rev Drug Discov 9:215-236.

Hosea NA, Collard WT, Cole S, Maurer TS, Fang RX, Jones H, Kakar SM, Nakai Y, Smith BJ, Webster R, et al. (2009) Prediction of human pharmacokinetics from preclinical information: comparative accuracy of quantitative prediction approaches. J Clin Pharmacol 49:513-533.

Hütter JF, Piper HM, and Spieckermann PG (1984a) Myocardial fatty acid oxidation: evidence for an albumin-receptor-mediated membrane transfer of fatty acids. Basic Res Cardiol 79:274-282.

Hütter JF, Piper HM, and Spieckermann PG (1984b) Kinetic analysis of myocardial fatty acid oxidation suggesting an albumin receptor mediated uptake process. J Mol Cell Cardiol 16: 219-226.

Jones HM, Barton HA, Lai Y, Bi YA, Kimoto E, Kempshall S, Tate SC, El-Kattan A, Houston JB, Galetin A, et al. (2012) Mechanistic pharmacokinetic modeling for the prediction of transportermediated disposition in humans from sandwich culture human hepatocyte data. Drug Metab Dispos 40:1007-1017.

Kalliokoski A and Niemi M (2009) Impact of OATP transporters on pharmacokinetics. $\mathrm{Br} \mathrm{J}$ Pharmacol 158:693-705.

Kim SJ, Lee KR, Miyauchi S, and Sugiyama Y (2019) Extrapolation of in vivo hepatic clearance from in vitro uptake clearance by suspended human hepatocytes for anionic drugs with high binding to human albumin: improvement of in vitro-to-in vivo extrapolation by considering the "albumin-mediated" hepatic uptake mechanism on the basis of the "facilitated-dissociation model". Drug Metab Dispos 47:94-103.

Lam JL and Benet LZ (2004) Hepatic microsome studies are insufficient to characterize in vivo hepatic metabolic clearance and metabolic drug-drug interactions: studies of digoxin metabolism in primary rat hepatocytes versus microsomes. Drug Metab Dispos 32:1311-1316.

Lam JL, Shugarts SB, Okochi H, and Benet LZ (2006) Elucidating the effect of final-day dosing of rifampin in induction studies on hepatic drug disposition and metabolism. J Pharmacol Exp Ther 319:864-870.

Lipitor (atorvastatin calcium). (1996) Package insert. Pfizer, New York, NY

Livalo (pitavastatin). (2009) Package insert. Kowa Pharmaceuticals America, Inc, Montgomery, AL.

Miyauchi S, Masuda M, Kim S-J, Tanaka Y, Lee K-R, Iwakado S, Nemoto M, Sasaki S, Shimono $\mathrm{K}$, Tanaka Y, et al. (2018) The phenomenon of albumin-mediated hepatic uptake of organic anion transport polypeptide substrates: prediction of the in vivo uptake clearance from the in vitro uptake by isolated hepatocytes using a facilitated-dissociation model. Drug Metab Dispos 46:259-267.

Nezasa K, Higaki K, Takeuchi M, Nakano M, and Koike M (2003) Uptake of rosuvastatin by isolated rat hepatocytes: comparison with pravastatin. Xenobiotica 33:379-388.

Nunes R, Kiang C-L, Sorrentino D, and Berk PD (1988) 'Albumin-receptor' uptake kinetics do not require an intact lobular architecture and are not specific for albumin. J Hepatol 7:293-304.

Obach RS (1999) Prediction of human clearance of twenty-nine drugs from hepatic microsomal intrinsic clearance data: an examination of in vitro half-life approach and nonspecific binding to microsomes. Drug Metab Dispos 27:1350-1359.
Pardridge WM, Sakiyama R, and Fierer G (1983) Transport of propranolol and lidocaine through the rat blood-brain barrier. Primary role of globulin-bound drug. J Clin Invest 71:900-908.

Poulin P, Bteich M, and Haddad S (2017) Supplemental analysis of the prediction of hepatic clearance of binary mixtures of bisphenol A and naproxen determined in an isolated perfused rat liver model to promote the understanding of potential albumin-facilitated hepatic uptake mechanism. J Pharm Sci 106:3207-3214.

Poulin P, Burczynski FJ, and Haddad S (2016) The role of extracellular binding proteins in the cellular uptake of drugs: impact on quantitative in vitro-to-in vivo extrapolations of toxicity and efficacy in physiologically based pharmacokinetic-pharmacodynamic research. J Pharm Sci 105:497-508.

Poulin P, Hop CECA, Ho Q, Halladay JS, Haddad S, and Kenny JR (2012) Comparative assessment of in vitro-in vivo extrapolation methods used for predicting hepatic metabolic clearance of drugs. I Pharm Sci 101:4308-4326.

Pravachol (pravastatin sodium). (1991) Package insert. Bristol-Myers Squibb, New York, NY.

Rauch B, Bode C, Piper HM, Hütter JF, Zimmermann R, Braunwell E, Hasselbach W, and Küble

W (1987) Palmitate uptake in calcium tolerant, adult rat myocardial single cells--evidence for an albumin mediated transport across sarcolemma. J Mol Cell Cardiol 19:159-166.

Shi JH, Wang Q, Pan DQ, Liu TT, and Jiang M (2017) Characterization of interactions of simvastatin, pravastatin, fluvastatin, and pitavastatin with bovine serum albumin: multiple spectroscopic and molecular docking. J Biomol Struct Dyn 35:1529-1546.

Shibata Y, Takahashi H, Chiba M, and Ishii Y (2002) Prediction of hepatic clearance and availability by cryopreserved human hepatocytes: an application of serum incubation method. Drug Metab Dispos 30:892-896.

Shibata Y, Takahashi H, and Ishii Y (2000) A convenient in vitro screening method for predicting in vivo drug metabolic clearance using isolated hepatocytes suspended in serum. Drug Metab Dispos 28:1518-1523.

Shimada S, Fujino H, Morikawa T, Moriyasu M, and Kojima J (2003) Uptake mechanism of pitavastatin, a new inhibitor of HMG-CoA reductase, in rat hepatocytes. Drug Metab Pharmacokinet 18:245-251.

Soars MG, McGinnity DF, Grime K, and Riley RJ (2007) The pivotal role of hepatocytes in drug discovery. Chem Biol Interact 168:2-15.

Sorrentino D, Robinson RB, Kiang C-L, and Berk PD (1989) At physiologic albumin/oleate concentrations oleate uptake by isolated hepatocytes, cardiac myocytes, and adipocytes is a saturable function of the unbound oleate concentration: uptake kinetics are consistent with the conventional theory. J Clin Invest 84:1325-1333.

Stollman YR, Gärtner U, Theilmann L, Ohmi N, and Wolkoff AW (1983) Hepatic bilirubin uptake in the isolated perfused rat liver is not facilitated by albumin binding. J Clin Invest 72:718-723.

Taft DR and Sweeney KR (1995) The influence of protein binding on the elimination of acetazolamide by the isolated perfused rat kidney: evidence of albumin-mediated tubular secretion. J Pharmacol Exp Ther 274:752-760.

Thomas DW, Burns J, Audette J, Carroll A, Dow-Hygelund C, and Hay M (2016) Clinical development success rates 2006-2015. BIO Industry Analysis 1-16.

Trainor GL (2007) The importance of plasma protein binding in drug discovery. Expert Opin Drug Discov 2:51-64.

Tsao SC, Sugiyama Y, Sawada Y, Iga T, and Hanano M (1988) Kinetic analysis of albuminmediated uptake of warfarin by perfused rat liver. J Pharmacokinet Biopharm 16:165-181.

Weisiger RA and Ma WL (1987) Uptake of oleate from albumin solutions by rat liver. Failure to detect catalysis of the dissociation of oleate from albumin by an albumin receptor. J Clin Invest 79:1070-1077.

Wood FL, Houston JB, and Hallifax D (2017) Clearance prediction methodology needs fundamental improvement: trends common to rat and human hepatocytes/microsomes and implications for experimental methodology. Drug Metab Dispos 45:1178-1188.

Yabe Y, Galetin A, and Houston JB (2011) Kinetic characterization of rat hepatic uptake of 16 actively transported drugs. Drug Metab Dispos 39:1808-1814.

Yamazaki M, Suzuki H, Hanano M, Tokui T, Komai T, and Sugiyama Y (1993) Na(+)independent multispecific anion transporter mediates active transport of pravastatin into rat liver. Am J Physiol 264:G36-G44.

Address correspondence to: Leslie Z. Benet, Department of Bioengineering and Therapeutic Sciences, University of California, 533 Parnassus Ave., Room U-68, Campus Box 0446, San Francisco, CA 94143-0912. E-mail: leslie.benet@ucsf.edu 